\title{
Classical Cesarean Section
}

\author{
Amano Kan, MD, $\mathrm{PhD}^{1}$ \\ 1 Department of Obstetrics and Gynecology, Center for Perinatal \\ Medicine, Kitasato University School of Medicine, Yoshida Obstetrics \\ and Gynecology Clinic, Tokyo, Japan \\ Surg J 2020;6(suppl S2):S98-S103.
}

Address for correspondence Amano Kan, MD, PhD, Department of Obstetrics and Gynecology, Center for Perinatal Medicine, Kitasato University School of Medicine, Yoshida Obstetrics and Gynecology Clinic, 251-0861 5061-4 Ohba Fujisawa-City, Kanagawa, Japan (e-mail: kanamano0101@gmail.com).

\begin{abstract}
Keywords

- cesarean section

- vertical uterine incision

- classical cesarean section

- uterine rupture

Cesarean section is the most frequent obstetric operation that is performed in cases when a vaginal delivery would put the fetus or mother at risk. Several procedures are offered depending on the indication and the degree of urgency. After laparotomy, the uterus can be incised by a variety of techniques, usually low transverse uterine incision is selected ( - Fig. 1). At times, a low transverse hysterotomy is selected but provides inadequate room for delivery. In such cases incision is extended such as Jextension, U-extension, and T-extension. However, in some cases, where the low transverse incision is arduous, a midline vertical incision (classical cesarean section) is considered.
\end{abstract}

Cesarean section is the most common surgery in obstetrics. Several techniques are proposed according to the indication and the degree of urgency. Usually laparotomy followed by hysterotomy with a low transverse incision is preferable. However, in cases in which it is difficult to access the lower uterine segment, such as that in preterm labor, dense adhesion, placenta previa/accrete a vertical hysterotomy (classical cesarean section) may be needed. Although a smooth and gentle delivery of the fetus is possible through the vertical incision, uterine closure is technically difficult. To decrease the risks of hemorrhage and adhesion, a speedy and skillful technique is mandatory. The most serious risk of vertical incision in the contractile corpus is uterine rupture in the subsequent pregnancy. Therefore, cases of prior classical cesarean section are contraindicated for trial of labor after cesarean section.

\section{Surgical Steps}

1. Midline vertical incision for laparotomy $\downarrow$

2. Hysterotomy by midline vertical incision above the lower segment $\downarrow$

3. Delivery of the fetus/placenta $\downarrow$

4. Uterine repair $\downarrow$

5. Abdominal closure

\section{Indications for Classical Cesarean Section}

\section{Preterm Labor}

Since the poorly developed lower uterine segment provides inadequate space for the manipulations required for fetal delivery, especially in cases prior to 30 weeks of gestation, with nonreassuring fetal status or inevitable preterm labor are candidates for the classical cesarean section. In instances when the fetus is very small, especially in case of a breech presentation, the small fetal head may become entrapped by the small low transverse incision space and uterine contractions, therefore classical cesarean section is desirable to prevent the fetal risk of intracranial hemorrhage. The risks of neonatal mortality and morbidity may be decreased by classical cesarean section in some cases of preterm labor (-Table 1).

\section{Fetal Indications}

Transverse lie of a large fetus, especially if the membranes are ruptured and the shoulder is impacted in the birth canal necessitates a classical incision. A fetus presenting as a backdown transverse lie is particularly difficult to deliver through a low transverse incision.

Malformed fetus such as conjoined twins, sacrococcygeal teratoma, macrocrania, myelomeningocele is difficult to deliver gently through a low transverse incision.
DOI https://doi.org/ 10.1055/s-0039-3402072. ISSN 2378-5128.
Copyright $\odot 2020$ by Thieme Medical Publishers, Inc., 333 Seventh Avenue, New York, NY 10001, USA. Tel: +1(212) 760-0888.
License terms

(๑) $\Theta \circledast$ 


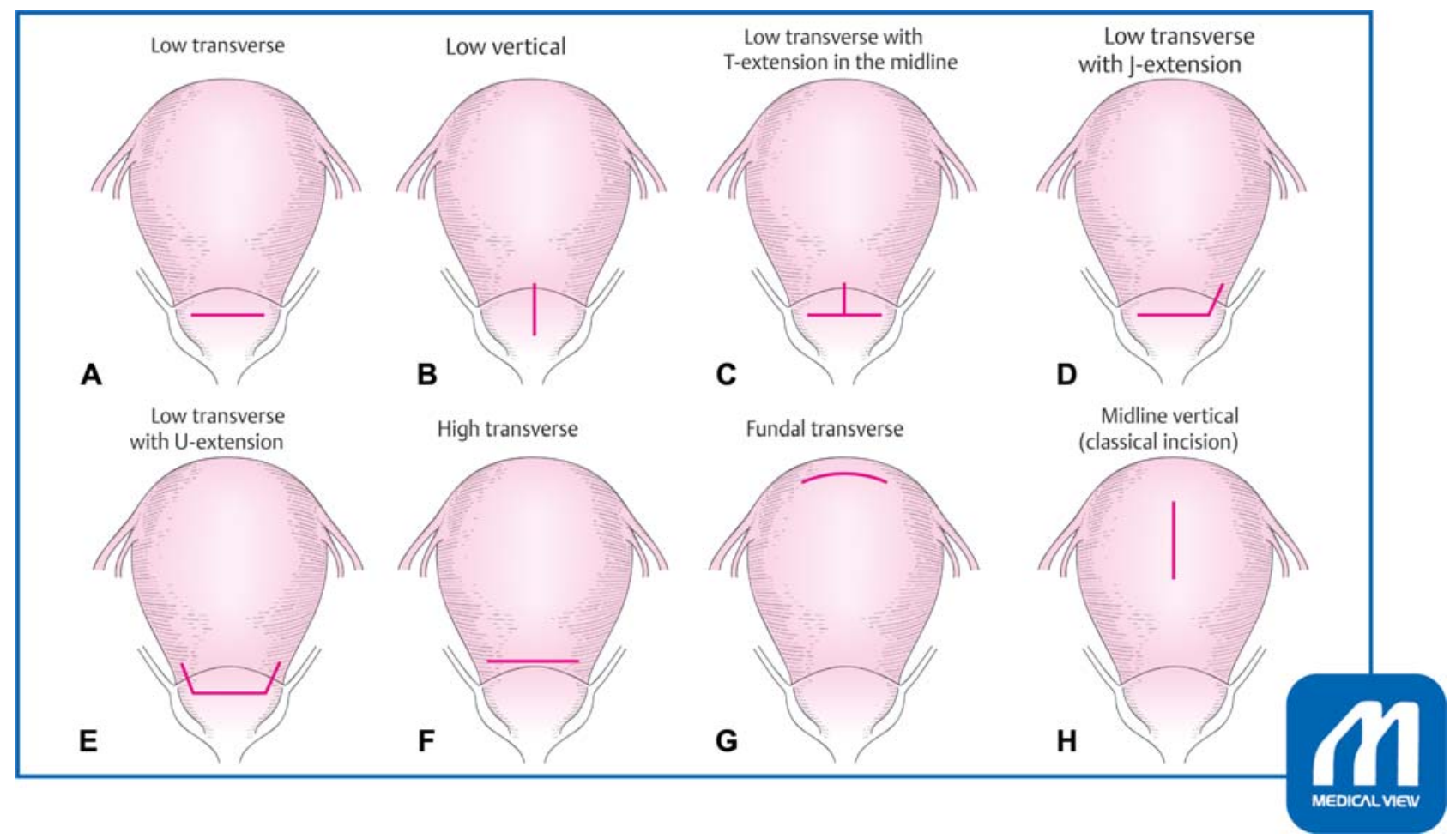

Fig. 1 Variety of incisions for hysterotomy. (A) Low transverse. (B) Low vertical. (C) Low transverse with T-extension in the midline. (D) Low transverse with J-extension. (E) Low transverse with U-extension. (F) High transverse. (G) Fundal transverse. (H) Midline vertical (classical incision). (Reproduced with permission of Amano K. In: Hiramatsu Y, Konishi I, Sakuragi N, Takeda S, eds. Mastering the Essential Surgical Procedures OGS NOW, No.3. Cesarean Section. (Japanese). Tokyo: Medical View; 2010:42-47. Copyright @ Medical View).

Table 1 Indications for classic cesarean section

\begin{tabular}{|c|c|}
\hline \multirow{2}{*}{$\begin{array}{l}\text { Preterm labor } \\
(\leq 30 \mathrm{wk})\end{array}$} & - Breech, transverse lie \\
\hline & - Nonreassuring fetal status \\
\hline \multirow{2}{*}{$\begin{array}{l}\text { Difficult to } \\
\text { access the } \\
\text { lower segment }\end{array}$} & $\begin{array}{l}\text { - Serious adhesion around the } \\
\text { vesicouterine space }\end{array}$ \\
\hline & - Morbid obesity \\
\hline \multirow{3}{*}{$\begin{array}{l}\text { Uterine } \\
\text { abnormality }\end{array}$} & - Myoma uteri \\
\hline & - Anomalous uteri \\
\hline & - Cervical carcinoma \\
\hline \multirow[t]{2}{*}{ Fetal indication } & $\begin{array}{l}\text { Malformation (macrocrania, } \\
\text { sacrococcygeal teratoma, } \\
\text { myelomeningocele, conjoined twins) }\end{array}$ \\
\hline & - Transverse lie \\
\hline
\end{tabular}

\section{Uterine Abnormality}

In cases of an anomalous uterus with a hypoplastic cervix, myoma uteri, or invasive cervical cancer a low transverse incision is not indicated.

\section{Difficult Access to the Uterine Lower Segment}

When it is very hard to access the uterine lower segment in cases with dense adhesion, or morbid obesity, incision into the vesicouterine peritoneum and separating the bladder is difficult, indicating a classical cesarean section.

\section{Placenta Previa}

In case of placenta previa, placental incision should be avoided, especially if the placenta accrete is suspected from prenatal ultrasonography and intraoperative inspection of the engorged uterine superficial vessels, a classical incision or a fundal transverse incision is advisable. If placenta accrete/increta is suspected, a uterine incision is performed while keeping away from the placenta and after delivering the fetus, the cord is ligated and cut, and the placenta is left in situ. The uterine incision is sutured by a continuous running locking suture, and a hysterectomy is immediately performed.

\section{Procedure of the Classical Cesarean Section}

\section{Abdominal Incision}

Usually a midline vertical incision is chosen for laparotomy.

A vertical infraumbilical incision provides quick entry to shorten the incision-to-delivery interval. Moreover, this incision has minimal blood loss, provides superior access to the upper abdomen and generous operating room, and offers flexibility for easy wound extension if greater space or access is needed. The main disadvantages are poorer cosmetic results, higher rates of fascial dehiscence or incisional hernia, and greater postoperative pain compared with a Pfannenstiel transverse incision.

An infraumbilical midline vertical incision begins 2 to $3 \mathrm{~cm}$ above the superior margin of the symphysis and should be of sufficient length $(12-14 \mathrm{~cm})$ to allow fetal delivery without difficulty. Sharp or electrosurgical dissection is 
performed to the anterior rectus sheath. Fascial incision is extended superiorly and inferiorly with scissors or scalpel. The rectus abdominis and pyramidalis muscles are subsequently separated, and the peritoneum is carefully opened. Before hysterotomy, the surgeon should palpate the fundus and adnexa to identify the degree of uterine rotation. The uterus may be dextrorotated due to the proximity of the sigmoid colon so that the left round ligament is more anterior and closer to the midline.

\section{Uterine Incision}

A midline vertical uterine incision in the contractile corpus is carefully initiated with a scalpel until the membranes appeared, and when the uterus is entered, the incision site is opened with fingers wide enough to make an adequate space to deliver the fetus. If the placenta is encountered in the incision line, the placenta is torn off and membranes are ruptured as quickly as possible to avoid severe fetal hemorrhage. As the incision is opened, numerous large vessels that bleed profusely are commonly encountered within the myometrium.

A speedy and skillful technique is mandatory.

A low vertical incision is made parallel to the longitudinal axis of the uterus in the midline with care being taken to stay below the contractile portion of the uterus and within the thin lower uterine segment. Studies have shown that there is no significant increased risk of uterine rupture in patients with this type of incision compared with low transverse incision.

\section{Delivery of the Fetus and Placenta}

After the membranes are ruptured, the fetus will be delivered easily compared with cases with a low transverse incision. The umbilical cord should be ligated and cut. Fundal massage may begin as soon as the fetus is delivered to hasten placental separation, and the placenta is manually removed. Immediately after delivery of the placenta, the uterine cavity is suctioned and wiped out with a gauze sponge to remove the remaining membranes, vernix, and clots.

After birth, to facilitate the uterine contraction, an intravenous infusion of 10 units oxytocin in $1 \mathrm{~L}$ of crystalloid solution may be begun. Second-line agents are ergot alkaloids, and the use of tranexamic acid has recently been described to lower blood loss during cesarean delivery.

There is insufficient evidence of mechanical or finger dilatation of the cervix during nonlabor cesarean section to reduce postoperative morbidity such as infection rates from potential hematometra.

\section{Uterine Repair}

After removal of the placenta, the uterus is lifted through the incision onto the abdominal wall. Although some clinicians prefer to avoid such exteriorization, there are often benefits that outweigh the disadvantages.

For incision closure, it is helpful to have an assistant compress the uterus on each side of the wound toward the midline as each stich is placed to achieve good approximation.

Because the classical incisions are much thicker, they are normally repaired in three layers.

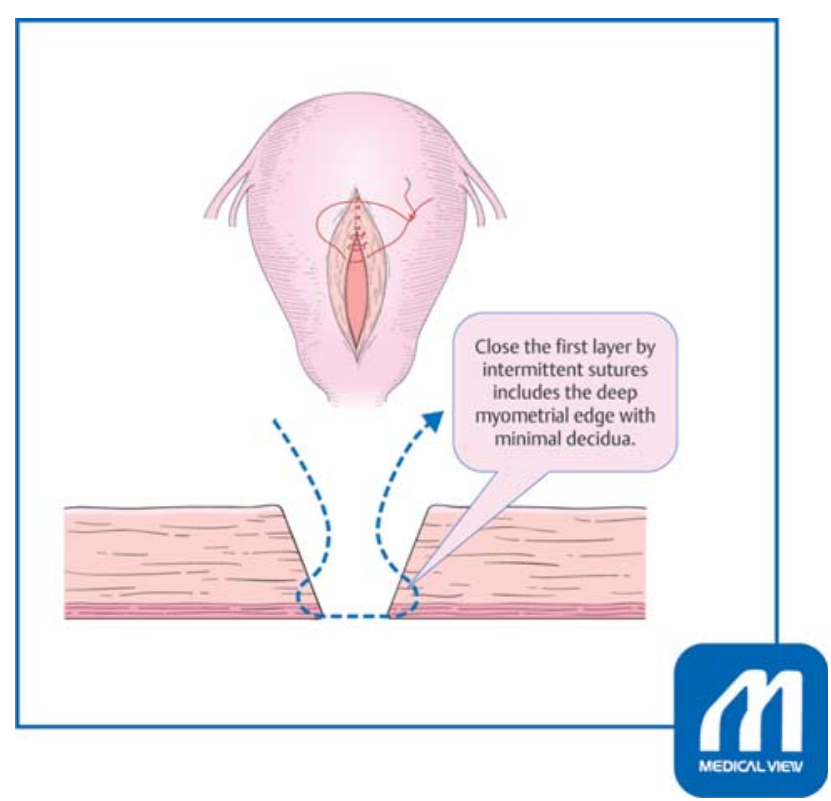

Fig. 2 Closure of the first layer. Text A: Closing the first layer by intermittent sutures includes the deep myometrial edge with minimal decidua. (Reproduced with permission of Amano K. In: Hiramatsu Y, Konishi I, Sakuragi N, Takeda S, eds. Mastering the Essential Surgical Procedures OGS NOW, No.3. Cesarean Section. (Japanese). Tokyo: Medical View; 2010:42-47. Copyright (c Medical View).

The main principle to remember is that the dead space needs to be obliterated to achieve hemostasis and it reduces the chance of hematoma formation. The first layer is closed with interrupted sutures (\#1 Coated VICRIL PLUS, ETICON Inc.) with decidual exclusion to avoid endometrial inversion at the scar site, because this may be the cause of incomplete scar healing (-Fig. 2). Concerns have been expressed that sutures through the decidua may lead to endometriosis or adenomyosis in the hysterotomy scar, however, this is rare. The second layer is also closed with interrupted sutures, and the final layer is closed with continuous locking sutures or figure-of-eight sutures (-Figs. 3, 4).

After closure of the incision, an adhesion barrier patch, such as SEPRAFILM, KAKEN Inc. or GYNECARE INTERCEED, ETICHON Inc. is applied.

\section{Abdominal Closure}

Prior to abdominal closure, all surgical sponges are removed, and the paracolic gutters and cul-de-sac are gently suctioned of blood and amniotic fluid. The uterine contraction, hemostasis of the incision, and the aspect of the adnexa are then confirmed. After gauze and instrument counts are found to be correct, the abdominal cavity is irrigated with warmed saline.

Abdominal incisions are closed in layers. Peritoneum fascia is closed with interrupted suture or continuous suture, and subcutaneous tissue is approximated with interrupted suture. Skin is closed with staplers and/or interrupted relaxation sutures. 


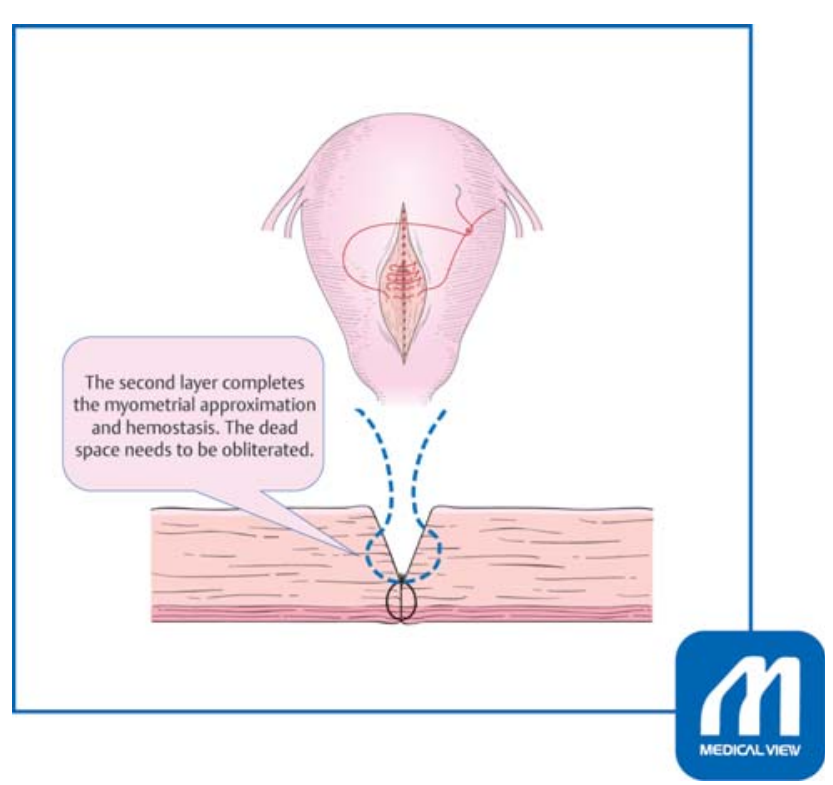

Fig. 3 Closure of the second layer. Text B: The second layer completes the myometrial approximation and hemostasis. The dead space needs to be obliterated. (Reproduced with permission of Amano K. In: Hiramatsu Y, Konishi I, Sakuragi N, Takeda S, eds. Mastering the Essential Surgical Procedures OGS NOW, No.3. Cesarean Section. (Japanese). Tokyo: Medical View; 2010:42-47. Copyright @ Medical View).

\section{Benefits and Risk of Classical Incision}

\section{Benefits}

The fetus can be delivered quickly and gently with minimal risk of forcing delivery which may result in intracranial hemorrhage in a preterm case.

Luthra et $\mathrm{al}^{1}$ compared the uterine incision-to-delivery interval and neonatal and maternal complications in vertical
Table 2 Merits and demerits for classical cesarean section

\begin{tabular}{|c|c|}
\hline \multirow[t]{3}{*}{ Merits } & - Deliver the fetus without difficulty \\
\hline & - Avoid bladder injury \\
\hline & - Extend incision without lacerating uterine arteries \\
\hline \multirow[t]{5}{*}{ Demerits } & - Increased blood loss \\
\hline & - Difficulty of uterine closure \\
\hline & - Increased complications of infection \\
\hline & - Postoperative adhesion \\
\hline & $\begin{array}{l}\text { - Subsequent uterine rupture or uterine scar } \\
\text { dehiscence }\end{array}$ \\
\hline
\end{tabular}

(low vertical; $n=53$, classical; $n=134$ ) versus low transverse incision in preterm cesarean section between 23 and 34 weeks of gestation. After adjusting for confounders, there was no significant difference in the incision-to-delivery interval between the two types of incisions. However, the risk for maternal transfusion was higher among those with a vertical incision. The incision type was not associated with any neonatal outcomes including intracranial hemorrhage, low Apgar score, or neonatal mortality. The need for rapid delivery is not justified by Luthra et als' findings, and this should no longer be considered as an indication for a vertical incision in the preterm population. Further studies including the effect on long-term outcome are warranted ( $\mathbf{- T a b l e ~} \mathbf{2}$ ).

\section{Risks}

Intraoperative, Postoperative Risk

As a classical uterine incision is made by incising the uterus parallel to the longitudinal axis of the uterus through the

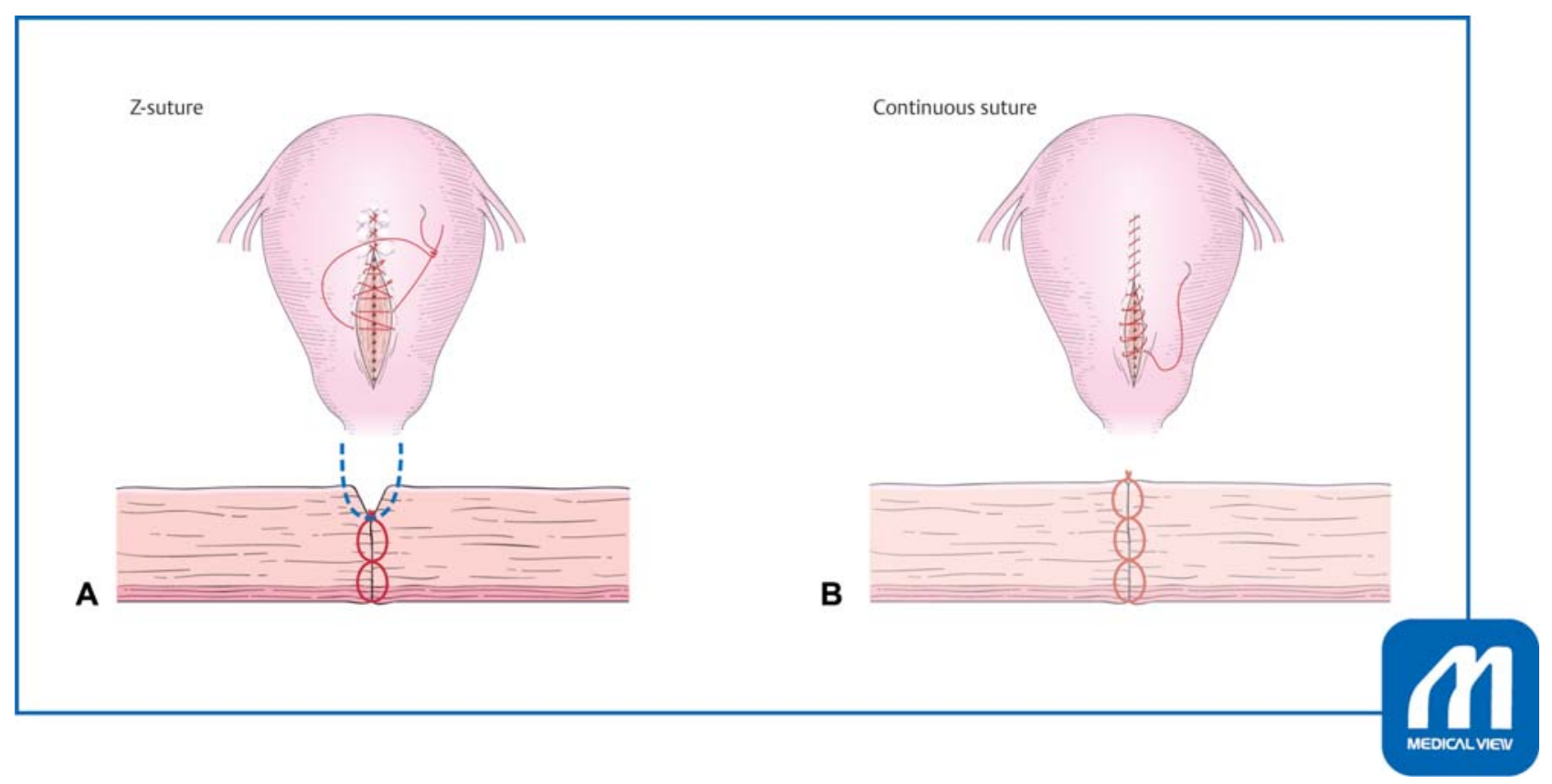

Fig. 4 Closure of the third layer. (A) Z suture. (B) Continuous suture, figure-of-eight suture. (Reproduced with permission of Amano K. In: Hiramatsu Y, Konishi I, Sakuragi N, Takeda S, eds. Mastering the Essential Surgical Procedures OGS NOW, No.3. Cesarean Section. (Japanese). Tokyo: Medical View; 2010:42-47. Copyright @ Medical View). 
contractile portion of the myometrium, so the bleeding from the myometrial vessels is excessive. Autologous blood transfusion should be provided for excessive bleeding in cases such as placenta previa, or uterine myoma. The most important thing to prevent excessive bleeding is prompt and skillful technique to approximate and suture of the incision site. The uterus is best managed with uterotonics and massaging the uterus while repairing the uterine incision. If the atony persists B-Lynch compression sutures can be used. Other options for hemorrhage control include bilateral uterine artery ligation at the level of the lower uterine segment and its anastomosis with the ovarian artery at the upper part of the uterus. Recently, interventional radiology is more commonly used. In patients who do not respond to these management strategies, hysterectomy is indicated for saving the mother.

Bladder injuries are extremely rare compared with cases with a low transverse incision.

Prevention of infection, deep vein thrombosis, and pulmonary embolism are considered in the same way as other types of cesarean sections. Early ambulation, elastic stockings, and intermittent pneumatic compression are indispensable. Anticoagulation prophylaxis is considered in cases with morbid obesity, history of thrombosis, and/or hemorrhagic diathesis.

Patterson et $\mathrm{al}^{2}$ estimated the maternal and perinatal morbidity associated with cesarean delivery involving 221 cases $(1.1 \%)$ of the classical incision compared with that of 19,422 cases $(98.5 \%)$ of the low transverse incision, and the inverted $\mathrm{T}$ incision in 83 cases (0.4\%). Maternal morbidity (puerperal infection, blood transfusion, hysterectomy, intensive care unit admission, and death) and perinatal morbidity (stillborn fetus, neonatal death, 5-minute Apgar less than 7 , and intensive care) were significantly higher with the classical incision compared with the low transverse incision. Some maternal morbidity (puerperal infection and blood transfusion) and perinatal morbidity (5-minute Apgar less than 7, and intensive care) were also significantly higher for the inverted $\mathrm{T}$ incision compared with the low transverse incision.

\section{Uterine Rupture}

\section{Case Presentation}

28 years old, G3-P1

Previous pregnancy course:

The patient was hospitalized at 22 weeks of gestation due to threatened preterm labor.

Preterm premature rupture of membranes occurred at 24 weeks and 6 days, and severe variable decelerations were frequently noted. Emergency cesarean section was performed under general anesthesia. Female baby, $562 \mathrm{~g}$ with Apgar score 3/5, UA-pH 7.17 was delivered through classical uterine incision. Neonatal death was unavoidable in spite of aggressive neonatal intensive care. For incision closure, the deeper half of the myometrium was approximated with intermittent sutures with \#1 Coated VICRIL PLUS, ETICON
Inc., and the outer depth of the myometrium was sutured with figure -of-eight sutures. Several Z-sutures were needed for hemostasis at the incision site. A GYNECARE INTERCEED patch was applied to prevent adhesions.

Current pregnancy course:

Her first medical examination was at 8 weeks of gestation; thereafter, her pregnancy course was uneventful. At 29 weeks and 4 days, she complained of abdominal distention. She was hospitalized and tocolytic treatment was offered with a prescription of a steroid for preterm labor. Elective cesarean section was scheduled at 34 weeks; however, the patient and her family desired to postpone the operation. She complained of severe abdominal pain with abrupt fetal bradycardia at 34 weeks and 5 days. Extremely emergent cesarean section was performed due to uterine scar rupture.

After laparotomy, bloody ascites was noted and the placenta was partially expelled through the ruptured incision site. A female baby, weighing 2,174 g with Apgar score 5\%, UA-pH 6.99, pCO2 $84 \mathrm{~mm} \mathrm{Hg}, \mathrm{BE}-13 \mathrm{mEq} / \mathrm{L}$ was delivered. The baby was admitted to neonatal intensive care unit, and her neonatal course was uneventful. The ruptured incision was restored with three layers of intermittent sutures.

The most serious complication of a classical cesarean incision is uterine rupture with subsequent pregnancy. In patients with a previous classical uterine incision, the risk of uterine rupture may be as high as 4 to $9 \%$, significantly higher than that of 0.2 to $1.5 \%$ with previous low transverse incision (-Table 3). ${ }^{3}$ In cases with a low vertical incision the incidence of scar disruptions and symptomatic ruptures is not increased compared with the cases with low transverse incision. ${ }^{4}$ Cases with a prior classical cesarean section are contraindicated for trial of labor after cesarean section (TOLAC).

Halperin et $\mathrm{l}^{5}$ studied the incidence of uterine rupture or uterine scar dehiscence among 326 women who had a primary preterm cesarean section. The classical incision was associated with a higher frequency of postpartum fever in the immediate postoperative period ( 16 vs. $6 \%, p<0.01$ ). Of the pregnancies after the classical operation $13 \%$ had abnormal scars compared with none of those after the low transverse operation $(p=0.0014)$. The frequency of scar dehiscence was $6 \%$ after a classical scar compared with none after a low transverse scar $(p=0.0581)$.

Moramarco et $\mathrm{al}^{6}$ studied the outcomes after preterm classical or low transverse caesarean section. Cases among those of 28 to 31-week gestation had increased risks of endometritis, transfusion, and ICU admission with the classical incision. They found that preterm classical caesarean section is not associated with significantly increased risks;

Table 3 Incidence of uterine rupture according to the type of uterine incision

\begin{tabular}{|l|l|}
\hline Classic incision & $4-9 \%$ \\
\hline Low transverse with T-extension & $4-9 \%$ \\
\hline Low vertical incision & $1-7 \%$ \\
\hline Low transverse & $0.2-1.5 \%$ \\
\hline
\end{tabular}

Source: ACOG, 1999. 
however, data are scarce. They concluded that subsequent uterine rupture risk when not planning a TOLAC is $1 \%{ }^{6}$

Among patients with prior classical cesarean delivery, uterine rupture or uterine scar dehiscence is neither predictable nor preventable; one-third of these occurring before the onset of clinical labor. ${ }^{7}$

Timing of the repeat cesarean section for patients who had undergone previous classical incision is determined considering the risks of uterine rupture compared with fetal prematurity. Gyamfi-Bannerman et $\mathrm{al}^{8}$ studied the risks of uterine rupture and placenta accrete in women with prior uterine surgery. Mean gestational age at delivery differed by groups, prior myomectomy ( $n=176,37.3$ weeks), prior classical cesarean section $(n=455,35.8$ weeks), and prior low transverse cesarean section ( $n=13,273,37.3$ weeks). The frequency of uterine rupture was not statistically different in cases with classical cesarean section $(0.88 \%)$ compared with low transverse cesarean section $(0.41 \%, p=0.13)$. The adjusted odds ratio for the classical cesarean section group (relative to low transverse groups) was 3.23 (95\% CI 1.11-9.39) for uterine rupture and 2.09 (95\% Cl 0.69-6.33) for placenta accrete.

A retrospective review ${ }^{9}$ by Chauhan et al included 37,863 deliveries and 157 patients (0.4\%) who underwent classical cesarean section. One case of uterine rupture $(0.6 \%)$ occurred at 29 weeks without preterm labor and resulted in fetal death. There was no significant difference between patients with uterine scar dehiscence $(n=15)$ and patients with intact uteri $(n=141)$ with regard to maternal demographics, duration of labor, cervical dilatation at the time of surgery, transfusion of packed red cells, bowel injury, postpartum endometritis, wound breakdown, thrombophlebitis, or umbilical pH $<7.00(p>0.05)$.

Poor predictors for uterine rupture or uterine scar dehiscence included duration of labor, cervical dilatation, and gestational age at repeat cesarean delivery. They concluded that one in four patients will likely experience some form of maternal morbidity. ${ }^{9}$

Stotland et $\mathrm{al}^{10}$ stated that the risks are lower in cases of elective cesarean section at 36 weeks of gestation without confirmation of fetal maturity compared with the cases of elective cesarean section at 39 weeks of gestation. The available data suggest that a scheduled cesarean section at 36 to 37 weeks optimizes both mother and fetal outcomes. ${ }^{11}$ Bakhshi et $\mathrm{al}^{12}$ studied the maternal and neonatal outcomes of repeat cesarean delivery at $\geq 36$ weeks. Outcomes were compared between cases with a previous classical cesarean section $(n=122)$ and those with a prior low transverse cesarean section $(n=7814)$. Uterine scar dehiscence was more frequent in cases of classical cesarean section (2.46 vs. $0.27 \%$, odds ratio $9.35,95 \% \mathrm{CI} 1.76-31.93$ ); however, no statistical differences in major maternal or neonatal morbidities between groups were noted.

Data from the NICHD MFMU Cesarean Registry (1999$2002)^{11}$ revealed that a total of $10 / 1051(0.095 \%)$ cases of uterine rupture in previous classical cesarean section were observed. Six hundred forty-three cases were delivered beyond 36 weeks with only three cases $(0.5 \%)$ of documented rupture. Noteworthy, seven ruptures occurred before 36 weeks, which would not have been prevented by a policy of scheduled delivery at 36 weeks.

To avoid such late preterm catastrophic uterine rupture, planned elective cesarean section at around 34 weeks may be considered, because the risk of severe neonatal morbidity is decreased dramatically after 34 weeks of gestation.

\section{Conflict of Interest}

None.

\section{References}

1 Luthra G, Gawade P, Starikov R, Markenson G. Uterine incision-todelivery interval and perinatal outcomes in transverse versus vertical incisions in preterm cesarean deliveries. J Matern Fetal Neonatal Med 2013;26(18):1788-1791

2 Patterson LS, O'Connell CM, Baskett TF. Maternal and perinatal morbidity associated with classic and inverted $\mathrm{T}$ cesarean incisions. Obstet Gynecol 2002;100(04):633-637

3 American College of Obstetricians and Gynecologists. ACOG practice bulletin. Vaginal birth after previous cesarean delivery. Number 5, July 1999 (replaces practice bulletin number 2, October 1998). Clinical management guidelines for obstetrician-gynecologists. Int J Gynaecol Obstet 1999;66(02):197-204

4 Shipp TD, Zelop CM, Repke JT, Cohen A, Caughey AB, Lieberman E. Intrapartum uterine rupture and dehiscence in patients with prior lower uterine segment vertical and transverse incisions. Obstet Gynecol 1999;94(5 Pt 1):735-740

5 Halperin ME, Moore DC, Hannah WJ. Classical versus low-segment transverse incision for preterm caesarean section: maternal complications and outcome of subsequent pregnancies. $\mathrm{Br} \mathrm{J}$ Obstet Gynaecol 1988;95(10):990-996

6 Moramarco V, Korale Liyanage S, Ninan K, Mukerji A, McDonald SD. Classical caesarean: what are the maternal and infant risks compared with low transverse caesarean in preterm birth, and subsequent uterine rupture risk? A systematic review and metaanalysis. J Obstet Gynaecol Can 2019:S1701-2163(19)30090-8

7 Scott JR. Avoiding labor problems during vaginal birth after cesarean delivery. Clin Obstet Gynecol 1997;40(03):533-541

8 Gyamfi-Bannerman C, Gilbert S, Landon MB, et al; Eunice Kennedy Shriver National Institute of Child Health and Human Development (NICHD) Maternal-Fetal Medicine Units (MFMU) Network. Risk of uterine rupture and placenta accreta with prior uterine surgery outside of the lower segment. Obstet Gynecol 2012;120(06):1332-1337

9 Chauhan SP, Magann EF, Wiggs CD, Barrilleaux PS, Martin JN Jr. Pregnancy after classic cesarean delivery. Obstet Gynecol 2002; 100(5 Pt 1):946-950

10 Stotland NE, Lipschitz LS, Caughey AB. Delivery strategies for women with a previous classic cesarean delivery: a decision analysis. Am J Obstet Gynecol 2002;187(05):1203-1208

11 Landon MB, Lynch CD. Optimal timing and mode of delivery after cesarean with previous classical incision or myomectomy: a review of the data. Semin Perinatol 2011;35(05):257-261

12 Bakhshi T, Landon MB, Lai Y, et al. Maternal and neonatal outcomes of repeat cesarean delivery in women with a prior classical versus low transverse uterine incision. Am J Perinatol 2010;27 (10):791-796 